\title{
Alterações oculares em crianças com toxoplasmose congênita precoce
}

\author{
Ocular manifestations in children with early Congenital Toxoplasmosis
}

\author{
Luciana Peixoto dos Santos ${ }^{(1)}$ \\ Lênio Souza Alvarenga ${ }^{(2)}$ \\ Magno Antônio Ferreira ${ }^{(3)}$
}

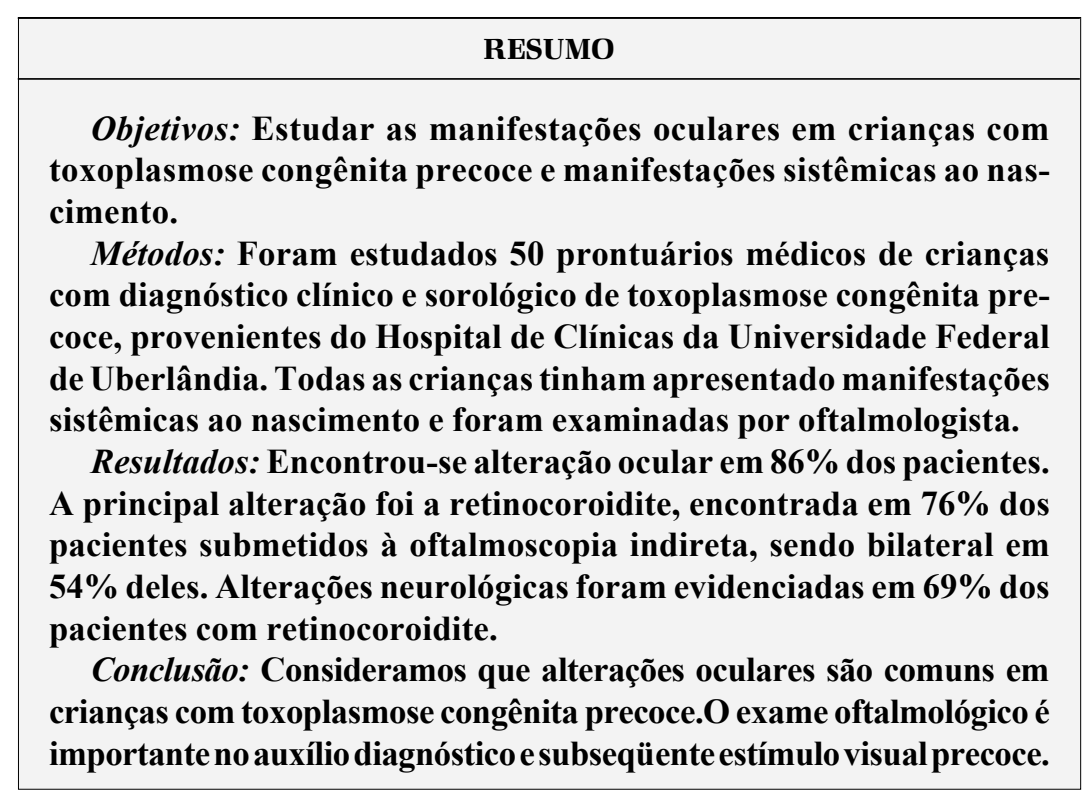

Palavras-chave: Toxoplasmose; Retinocoroidite; Manifestações sistêmicas
Apresentação em congresso : XII Congresso Brasileiro de Prevenção de Cegueira- São Paulo-SP-1996 Departamento de Oftalmologia da Universidade Federal de Uberlândia - UFU

(1) Graduação em medicina pela Universidade Federal de Uberlândia - UFU; Residente em Oftalmologia do terceiro ano na Universidade Federal de São PauloUNIFESP.

(2) Graduação em medicina pela Universidade Federal de Uberlândia - UFU; Residente em Oftalmologia do segundo ano na Universidade Federal de São PauloUNIFESP.

(3) Mestre em Oftalmologia pela Universidade Federal de São Paulo-UNIFESP; Departamento de Oftalmologia da Universidade Federal de Uberlândia.

Endereço para correspondência: Luciana Peixoto dos Santos. Rua Canário, 988, apto 132. São Paulo(SP) CEP 04521-004.E-mail:Lucpeixoto@oftalmo.epm.br

\section{INTRODUÇÃ̃O}

A toxoplasmose congênita é considerada importante causa mundial de morbidade e mortalidade infantis. Estima-se que nasçam anualmente no Brasil, cerca de 60.000 crianças com a doença, sendo portanto de alta magnitude ${ }^{1}$. A forma clínica em que somente os olhos são afetados é a maneira mais comum de manifestação da doença, além de ser a causa mais freqüente de uveíte no Brasil e importante causa evitável de cegueira ${ }^{1-4}$.

Classicamente, desde 1973 já relatado por Perkins ${ }^{5}$ e outros ${ }^{6}$, a doença ocular normalmente representa manifestação tardia de infecção congênita. A maioria dos casos são assintomáticas e estudos anteriores mostram uma escassez de lesões oculares ao nascimento e um aumento na prevalência das mesmas durante a adolescência ${ }^{7}$, predominando em jovens entre a segunda e terceira década ${ }^{1}$.

Das manifestações oculares descritas, a retinocoroidite é a mais comum, sendo que alguns a consideram o sinal mais freqüente da toxoplasmose congênita ${ }^{1}$. Alguns estudos mostram uma maior frequência e gravidade de lesões oculares em crianças com toxoplasmose congênita severa ao nascimento, muitas vezes, associadas a uma severidade maior nas lesões de sistema nervoso ${ }^{8}$.

O presente estudo se propõe a verificar a incidência de alterações 
oculares precoces em crianças portadoras de toxoplasmose congênita com manifestações sistêmicas ao nascimento.

\section{MATERIAL E MÉTODO}

Realizamos estudo retrospectivo, através de prontuário médico, de 50 crianças do Setor de Pediatria do Hospital de Clínicas da Universidade Federal de Uberlândia - HC-UFU, com diagnóstico clínico e sorológico, ao nascimento, de toxoplasmose congênita e que haviam sido submetidas à avaliação oftalmológica.

Foram consideradas portadoras de toxoplasmose congênita as crianças que preenchessem os seguintes requisitos ${ }^{1}$ :

- IgM específico positivo (Imunofluorescência Indireta ou Elisa)

- IgG específico positivo associado à quadro clínico suspeito e confirmado com nova sorologia após o $6^{\circ}$ mês de vida

- IgG específico positivo após o $6^{\circ}$ mês, com títulos estáveis ou maiores em relação ao nascimento.

Aquelas crianças que apresentaram outras infecções congênitas associadas, bem como aquelas sem manifestações clínicas ao nascimento foram exclusas do trabalho.

\section{RESULTADOS}

O estudo constou de 50 pacientes, sendo 27 (54\%) do sexo feminino e $23(46 \%)$ do sexo masculino. A média de idade por ocasião do primeiro exame oftalmológico foi de 3,2 meses, variando de 2 dias a 7 meses.

Dos 50 pacientes estudados, 43 (86\%) apresentaram alguma manifestação oftalmológica. A fundoscopia foi realizada em 34 (68\%) pacientes. Nos demais pacientes a avaliação do Fundo de Olho (F.O.) foi prejudicada devido à opacidade dos meios dióptricos.

A alteração ocular mais comum foi a retinocoroidite (RC), observada em 26 pacientes, o que representa $52 \%$ do total de casos ou $76 \%$ do número de fundoscopias realizadas. A lesão era bilateral em 14 pacientes (54\%).

As outras manifestações mais encontradas foram: Estrabismo $(32 \%)$, microftalmia (28\%), nistagmo $(28 \%)$, catarata (24\%) e opacidade vítrea (12\%) (gráfico 1). Outros achados menos freqüentes como palidez de papila ( 3 casos), sinéquias posteriores ( 3 casos) e descolamento de retina bilateral (1 caso) foram também observados.

Em relação à distribuição topográfica da retinocoroidite, em 21 pacientes $(81 \%)$ se localizou no pólo posterior, dos quais 19 (90\%), na região macular (gráfico 2). A mácula de ambos os olhos foi afetada em 11 pacientes, o que corresponde à $42 \%$ do total de casos de RC.

Dos pacientes com RC, 18 (69\%) apresentaram manifestações neurológicas tais como calcificações cerebrais, microencefalia, convulsões, hidrocefalia e alterações liquóricas.Três pacientes sem retinocoroidite $(37 \%)$ apresentavam alguma manifestação neurológica (gráficos 3 e 4).
Observamos uma associação entre estrabismo e nistagmo com RC. Dos pacientes com estrabismo (16 casos ), 87\%(14) apresentaram RC; dos pacientes com nistagmo (14 casos), $78 \%$ (11) apresentaram essa lesão. Catarata não esteve associada a nenhum caso de estrabismo ou nistagmo.

\section{DISCUSSÃO}

Observamos que todos os nossos casos foram diagnosticados no primeiro ano de vida, provavelmente porque, nossa amostra, conste de pacientes que apresentaram manifestações clínicas ao nascimento. Outros estudos mostram uma variação de $35-70 \%$ de diagnóstico nesta faixa etária ${ }^{1}$.

Não houve diferença significativa em relação ao sexo, reafirmando que a doença não tem afinidade especial por um ou outro sexo ${ }^{1}$.

A RC foi a alteração ocular mais comum em nossa amostra, o que está de acordo com a literatura ${ }^{1,2,8-12}$. Estudos que abrangem pacientes maiores de 1 ano apresentam freqüência maior de lesões retinianas, explicado pelo fato de que essas lesões seriam, provavelmente, de aparecimento tardio ${ }^{5,7} \mathrm{e}$ também pela maior facilidade de execução do exame de fundo de olho (FO) nessa faixa etária. Se considerarmos que $76 \%$ das crianças nas quais a fundoscopia foi viável apresentaram $\mathrm{RC}$, teremos uma freqüência de lesões comparável às crianças com maior idade. Outros estudos que, como o nosso, abrangeram apenas pacientes com toxoplasmose congênita severa ao nascimento, encontraram $100 \%$ de casos de RC ${ }^{8,11}$. Assim

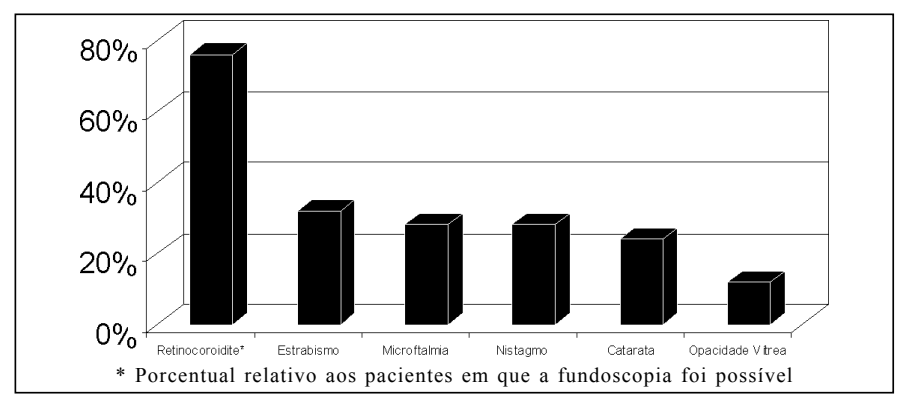

Gráfico 1 - Manifestrações oculares em crianças com toxoplasmose congênita precoce com manifestações sistêmicas ao nascimento

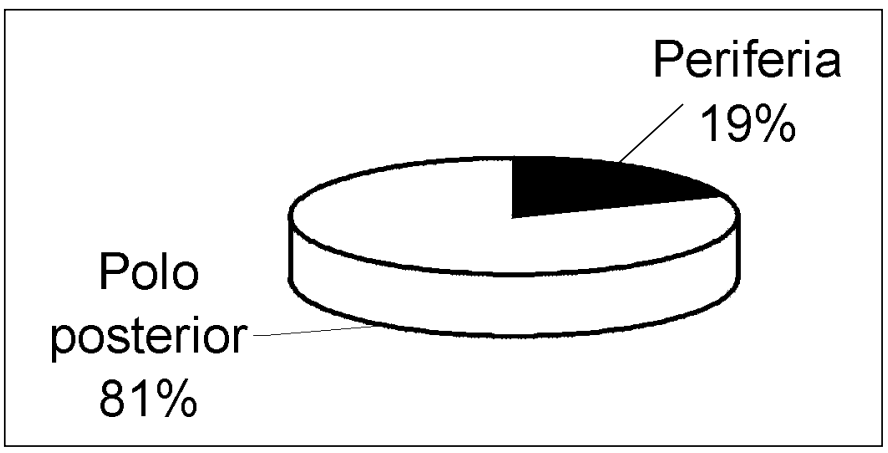

Gráfico 2 - Distribuição topográfica da retinocoroidite 


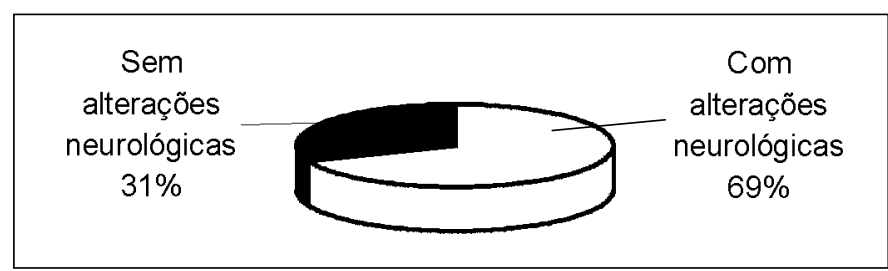

Gráfico 3 - Presença de alterações neurológicas nas crianças que apresentavam retinocoroidite

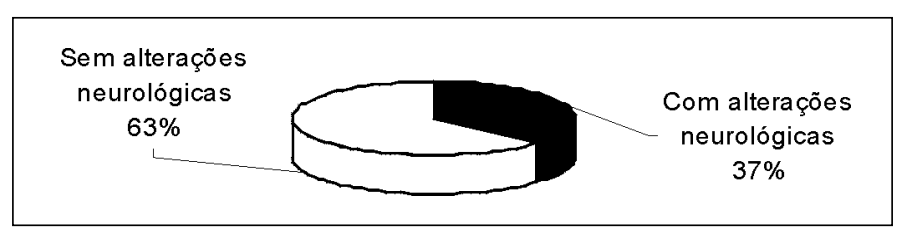

Gráfico 4 - Presença de alterações neurológicas nas crianças que não a presentavam retinocoroidite

como na Tétrade de Sabin, é possível que as lesões de retinocoroidite sejam de aparecimento mais precoce naquelas crianças com manifestações clínicas sistêmicas ao nascimento, como resultado de uma reação generalizada do parasita contra o hospedeiro.

A RC foi bilateral em $54 \%$ dos casos. A maioria dos autores consideram as lesões típicas da toxoplasmose congênita como sendo bilaterais $1,3,10,13$.

Associação de RC com lesões do sistema nervoso central é relatada em muitos trabalhos ${ }^{3,8,11}$. Em nosso estudo, $69 \%$ dos pacientes com lesão retiniana tinham manifestações neurológicas contra $37 \%$ nos pacientes sem retinocoroidite; essa diferença não foi estatisticamente significante, embora a análise estatística pelo teste de McNemar sugira uma correlação entre essas duas variáveis. Isso nos chama atenção para o fato de que, provavelmente, o $T$. gondii tenha um tropismo especial pelo sistema nervoso, já que a retina representa extensão do mesmo. Outros estudos mostraram que, um comprometimento ocular mais severo reflete comprometimento neurológico mais intenso, o que ocorreria em casos de exposição ao parasita em fases precoces da gestação ${ }^{8}$.

Em relação à topografia da lesão, tivemos um alta incidência de lesões no pólo posterior ( $81 \%$ ), dos quais $90 \%$ na região macular. O comprometimento macular bilateral, observado em $42 \%$ do total de casos de RC, representa um sério comprometimento da função visual nessas crianças. Sabe-se que a mácula é a localização preferencial da toxoplasmose congênita de manifestação precoce, por razões não esclarecidas ${ }^{1,13}$.

Dos outros achados mais encontrados, chamamos atenção em relação a uma provável associação de estrabismo e nistagmo com RC, o que ocorreria pela baixa acuidade visual causada pelas lesões retinianas centrais ${ }^{1}$. O achado de 12 casos $(24 \%)$ de catarata reforça mais ainda a importância de diagnóstico precoce nessas crianças, já que representa uma das principais causas recuperáveis de cegueira infantil ${ }^{14}$.
Outros estudos mostram até $40 \%$ de catarata associada à toxoplasmose congênita severa ${ }^{11}$; estudo anterior ${ }^{15}$, que avaliou a freqüência etiológica de catarata congênita, encontrou $2 \%$ de casos relacionados à toxoplasmose, porém esse estudo, diferente do nosso, não incluiu apenas casos graves da doença. A presença de nistagmo foi maior em crianças sem catarata, embora não tenha sido estatisticamente significante. Em nosso estudo, o não desenvolvimento de nistagmo nas crianças com catarata, provavelmente se deveu ao fato do exame ter sido realizado nos primeiros meses de vida onde se dá o período crítico para o desenvolvimento do reflexo de fixação ${ }^{16}$. As demais alterações oculares encontradas são todas descritas ${ }^{8,10-12}$.

Diante das inúmeras alterações oftalmológicas possíveis e da facilidade do exame, consideramos a avaliação oftalmológica de fundamental importância no diagnóstico de toxoplasmose congênita. O exame oftalmológico deve ser feito de rotina em todas as crianças com quadro clínico suspeito dessa infecção e repetidos posteriormente, a fim de se evidenciar as lesões típicas dessa doença. Por ser uma doença de alta freqüência, causando lesões de caráter irreversível e com graves seqüelas visuais, reforçamos também a importância da estimulação visual precoce e prevenção da infecção congênita, o que contribuiria profundamente com a diminuição do número de casos evitáveis de cegueira em crianças e adultos jovens.

\section{SUMMARY}

Purpose: To describe the ocular findings in children with early toxoplasmosis and sistemic manifestation of the disease

Methods: Fifty children with early congenital toxoplasmosis were included in this study and all data collected from their medical charts from the Hospital de Clínicas de Uberlândia. These children had their diagnosis based on clinical features and serological tests. All of them presented with systemic manifestations at birth and were examined by an ophthalmologist.

Results: Ocular manifestations were present in $86 \%$ of the patients. Retinochoroiditis was the most frequent lesion and was present in $76 \%$ of the patients that underwent indirect ophthalmoscopy, being bilateral in 54\% of them. Neurological findings were seen in 69\% of the patients with retinochoroiditis.

Conclusion: The authors consider that ocular manifestations are common in children with early congenital toxoplasmosis showing systemic manifestations at birth. Ocular examination should be performed in all suspected or confirmed cases due to its important help not only in the diagnosis but also because it leads to early visual stimulation.

Keywords: Toxoplasmosis; Retinochoroiditis; Systemic alterations. 


\section{REFERÊNCIAS BIBLIOGRÁFICAS}

1. Bahia MD, Oréfice F, Andrade GMQ. Análise clínica das lesões de retinocoroidite em crianças portadoras de toxoplasmose congênita. Rev Bras Oftal 1992;51(5):266-71.

2. Abreu MT, Hirata PS, Belfort Jr R, Domingues S. Uveítes em São Paulo: estudo epidemiológico, clínico e terapêutico, Arq Bras Oftalmol 1980;43:10-6.

3. Abreu MT, Belfort Jr R, Oréfice F. Toxoplasmose, In: Uveítes, Fernando Oréfice e Rubens Belfort Jr. Ed Roca 1987;21:212-30.

4. Fernandes LC, Oréfice F. Aspectos clínicos e epidemiológicos das uveítes em Serviços de Referência em Belo Horizonte de 1970 a 1993. Rev Bras Oftalmol 1996;55:569-78.

5. Perkins ES. Ocular Toxoplasmosis. BR J Ophtalmol 1973;57:1

6. Pinheiro SRA, Oréfice F, Andrade GMQ, Caialla WT. Estudo da toxoplasmose ocular em famílias de pacientes portadores de toxoplasmose congênita, sistêmica e ocular. Arq Bras Oftalmol 1990;53:4-6.

7. Koppe JG, Louwer - Sieger DH, Roever-Bonnet H. Results of 20 years followup of congenital toxoplasmosis. Lancet 1986;1:254-6.

8. O Neill JF. The ocular manifestations of congenital infection: a study of the early effect and long-term outcome of maternally transmitted rubella and toxoplasmosis. Trans Am Ophthalmol Soc 1998;96:813-79.

9. Martins CM, Silveira CM, Jamra LF, Barros PM, Belfort Jr R, Rigueiro MP Neves RA. Isolamento de Toxoplasma gondii de carnes e derivados, provenientes de região endêmica de Toxoplasmose ocular - Erechin - RS Arq Bras Oftalmol 1990;53(2):60-6.

10. Mets MB, Holfels E, Boyer KM, McLeod R. Eye manifestations of congenital toxoplasmosis. Am J Ophthalmol 1997 Jan;123(1):1-16.

11. Meenken C, Assies J, van Nieuwenhuizen O, Rothova A. Long term ocular and neurological involvement in severe congenital toxoplasmosis. Br J Ophthalmol 1995;79(6):581-4

12. Jankowska H, Szczepanska-Putz M. Observations of children with chorioretinitis in congenital toxoplasmosis. Pol Tyg Lek 1992 Mar 2-9;47(9-10):232-3.

13. Rodrigues MLV. Uveites. In: Abreu MT, Belfort Jr R, Oréfice F. Oftalmologia clínica. 1a. ed, Rio de Janeiro, Cultura médica 1992;171-203.

14. Taylor DSI, Rice NSC. Congenital cataract - a cause of preventable child blindness. Arch Dis Childhood 1982:57:165.

15. Kitadai SPS. Catarata Congênita - Frequência etiológica. Arq Bras Oftal $1992 ; 57(6): 404-6$

16. Parks M. Visual results in aphakic children. Am J Ophthalmol 1982;94:441.

\section{$2^{\circ}$ CONGRESSO DE OFTALMOLOGIA - USP 26 a 28 de Novembro de 1999 Centro de Convenç̃oes Rebouças - São Paulo - SP}

\section{SIMPÓSIOS}

Catarata / Refração: 0 que o oftalmologista precisa saber sobre óptica / Glaucoma / Ceratites LASIK - Complicações: prevenção e conduta

\section{URSOS}

Doencas da mácula / Estrabismo /Conjuntivites crônicas /Cirurgia de conjuntiva

Bases farmacológicas da terapêutica / Anamnese e exames complementares em doenças externas

Biomicroscopia / Ecografia / Retina periférica: orientações práticas para o oftalmologista geral

Doencas vasculares da retina / Retina cirúrgica / Uveítes / Neuroftalmologia / Lentes de contato Plástica ocular / LASIK para oftalmologista geral / Administração de consultório / Pesquisa científica

Visão subnormal / Campo visual computadorizado

\section{$1^{\circ}$ CONGRESSO DE AUXILIAR DE OFTALMOLOGIA}

CURSO I - "Visão subnormal"

CURSO II - "Básico para Auxiliar de Oftalmologia"

CURSO III- "Como evitar infeccão no consultório"

CURSO IV - "Laser em oftalmologia"

CURSO V. "Como realizar testes diagnósticos específicos"
CURSO VI - "Como organizar o setor de lentes de contato"

CURSO VII - "Auxiliar no centro cirúrgico"

CURSO VIII- "Administracão de consultório"

CURSO IX - "Campo visual"

\section{ATIVIDADES}

\section{PRÉ-CONGRESSO}

De 22 a $25 / 11$ - Wet Lab de Facoemulsificação

\section{ATIVIDADES PARALELAS}

Wet Lab de Visão Subnormal / Wet Lab de Lente de Contato / Wet Lab de Refração e Ótica

\section{N F O R M A C ÕE S}

CBO EVENTOS - Al. Santos, $1343 / 1110$ - $01419-001$ - São Paulo - SP

Tel: +11 3266-4000 / Fax: +11 3171-0948 / Email: eventos@cbo.com.br 